\title{
Vascular endothelial growth factor plays a critical role in the formation of the pre-metastatic niche via prostaglandin E2
}

\author{
SHILI LIU ${ }^{2}$, MAN JIANG ${ }^{1}$, QIANG ZHAO ${ }^{2}$, SHANCHENG LI ${ }^{1}$, YANPING PENG ${ }^{2}$, \\ PENGFEI ZHANG ${ }^{1}$ and MINGYONG HAN ${ }^{1}$ \\ ${ }^{1}$ Cancer Therapy and Research Center, Shandong Provincial Hospital, Shandong University, Jinan, Shandong 250021; \\ ${ }^{2}$ Department of Medical Microbiology, School of Medicine, Shandong University, Jinan, Shandong 250012, P.R. China
}

Received July 9, 2014; Accepted September 1, 2014

DOI: $10.3892 /$ or.2014.3516

\begin{abstract}
Factors secreted by primary tumors can alter the microenvironment at distant organ sites, generating pre-metastatic niches for subsequent metastatic cancer cell colonization. Breast cancer cells have a propensity to home preferentially to the lung, but the underlying molecular mechanisms whereby primary breast carcinoma-derived factors affect the premetastatic lung environment before the arrival of the tumor cells are poorly understood. In this study, 4T1 mammary carcinoma cells were subcutaneously injected into the mammary glands of mice, resulting in the induction of inflammation, increased total vessel density and recruitment of bone marrow-derived cells (BMDCs) in pre-metastatic lungs. Subsequent examination revealed that the sites of inflammatory cell clusters in the lungs were tumor metastasis sites. Moreover, vascular endothelial growth factor (VEGF) induced prostaglandin E2 (PGE2) production in mouse pulmonary microvascular endothelial cells (MPVECs) and enhanced the adhesion of 4T1 cells. Treatment with the cyclooxygenase- 2 inhibitor celecoxib significantly reduced 4T1 cell adhesion to MPVECs, and also reduced cancer metastasis and the inflammatory response. These results suggest that VEGF may be an underlying carcinoma-derived factor responsible for formation of the pre-metastatic niche in the lung of 4T1 cell-bearing mice. This study, therefore, demonstrated that primary tumors can alter the lung microenvironment during the pre-metastatic phase by triggering an inflammatory response and PGE2 production. Primary tumor-derived VEGF might thus be a crucial factor responsible for the formation of the pre-metastatic niche by inducing PGE2 production.
\end{abstract}

Correspondence to: Dr Mingyong Han, Cancer Therapy and Research Center, Shandong Provincial Hospital, Shandong University, 324 Jingwuweiqi Road, Jinan, Shandong 250021, P.R. China

E-mail: hanmingyong@hotmail.com

Key words: pre-metastatic niche, molecular mechanism, cancer metastasis, vascular endothelial growth factor, prostaglandin E2

\section{Introduction}

During cancer metastasis, tumor cells detach from their neighbors, invade the surrounding stroma and undergo intravasation. They survive in the circulation and arrest in the vessels of the target organ where they undergo extravasation, invade the matrix, and proliferate within the organ parenchyma to complete the metastatic process (1-3). However, reaching another location in the body does not guarantee that a metastatic tumor will form, and successful colonization and proliferation of tumor cells depends on the interaction of the cells with the microenvironment at the site of metastasis.

Previous studies found that the suitability of a site for metastatic colonization was determined not only by the characteristics of cancer cells, but also by the local microenvironment (1,4-10). Hematopoietic progenitor cells and myeloid cells were reportedly recruited to the lung by primary tumors to form the microenvironment. Furthermore, emerging evidence has revealed that bone marrow-derived cells (BMDCs) are a major player in the formation of pre-metastatic niches $(1,8)$.

The lung is a common target site for cancer cell metastasis. Recent reports showed that the lung microenvironment was modulated by distant primary tumors before the metastatic cancer cells arrived. This process occurred through changes in the lung in response to factors secreted by the primary tumors.

Considering the key role of the microenvironment in tumor metastasis, there has been speculation regarding the possible molecular processes whereby the primary tumor modulates the pre-metastatic niche in the lung prior to the formation of metastasis. Although several molecules have been implicated $(11,12)$, such as matrix metalloproteinase-9, S100A8 and S100A9, the mechanisms underlying lung microenvironmental changes in the pre-metastatic phase remain largely unknown.

In the present study, we investigated the effects of the primary tumor on the lung microenvironment, and explored the role of tumor-derived vascular endothelial growth factor (VEGF) as a key initiator of pre-metastatic modulation, and its relationship with prostaglandin E2 (PGE2) production.

\section{Materials and methods}

Cells and reagents. 4T1 cells were cultured in RPMI-1640 with $10 \%$ fetal calf serum medium. Six-week-old female 
Balb/c mice were acclimated and caged in groups of 6 or less. Celecoxib was manufactured by Pharmacia (Peapack, NJ, USA), rVEGF was purchased from Roche Applied Science (Basel, Switzerland) and a rat anti-mouse CD31 monoclonal antibody was from BD-Pharmingen. Celecoxib was fed on diet after 14 days of tumor cells implanted on the back of the mice, rVEGF was intraperitoneally injected daily at a dose of $5 \mathrm{mg} / \mathrm{kg}$.

Bone marrow transplantation. All animal studies were reviewed and approved by the Animal Care and Use Committee of the Shandong Provincial Animal Board (Jinan, China). Mice (6-8 weeks old) labeled with enhanced green fluorescent protein (EGFP) were used as donors of bone marrow (BM). BM cells (BMCs) were prepared according to procedures previously described (1). BMDCs were flushed out with RPMI-1640 medium from femurs and tibiae using 21-gauge needles. Then cells were harvested by centrifugation, resuspended with phosphate-buffered saline (PBS), and intravenously injected at a total number of $1 \times 10^{6}$ cells per $100 \mu \mathrm{l}$ PBS into recipient syngenic Balb/c mice that had received whole body irradiation of $900 \mathrm{rad}$ immediately before the transplantation.

$R N A$ extraction and $R T-q P C R$. Total RNA was extracted from PBS perfused lungs with the RNeasy kit (Qiagen) according to the manufacturer's protocol. First-strand cDNA was synthesized from $1 \mu \mathrm{g}$ total tissue RNA using the RevertAid ${ }^{\mathrm{TM}}$ First Strand cDNA synthesis kit (Thermo Fisher Scientific, Waltham, MA, USA) with random primers. Then cDNA was amplified for quantitative real-time PCR. The specific primers used were: Cox-2, forward, 5'-ATCAGGTCATTGGTGGAG-3' and reverse, 5'-ACACTCTGTTGTGCTCCC-3'; $\beta$-actin, forward, 5'-CTGTCCCTGTATGCCTCTG-3' and reverse, 5'-ATGTCACGCACGATTTCC-3'. Real-time PCR reactions were performed at: $95^{\circ} \mathrm{C}, 10 \mathrm{sec}$ (denaturation); $55^{\circ} \mathrm{C}, 30 \mathrm{sec}$ (annealing); $72^{\circ} \mathrm{C}, 30 \mathrm{sec}$ (extension) for 35 cycles. Real-time PCR reactions were performed on the ABI 7000 Fast real-time PCR system with SYBR-Premix Ex Taq ${ }^{\mathrm{TM}}$ according to the manufacturer's instructions.

Microarray analysis of the lungs. Balb/c mice were injected with 4T1 cells; control mice received PBS only. Twelve days after tumor-cell inoculation, mice were euthanized and lungs were perfused with PBS to completely remove blood from the lung vasculature. Cleaned lung lobes were immersed in RNAlater $^{\mathrm{TM}}$ to stabilize RNA. Total RNA was isolated and used for subsequent microarray analysis. Affymetrix Gene Chip mouse genome 4302.0 array was applied to analyze the expression profile of tissue samples. The microarray chip consisting of 27,326 different human cDNAs (Angilent, Wilmington, DE, USA) was employed to the analysis of the MPVECs treated with lipopolysacharide (LPS) and VEGF.

Cancer cell labeling and in vivo homing assay. 4T1 cells were labeled with DiI, then $2 \times 10^{4}$ labeled cells were infused i.v. In mice with primary tumors, the labeled cells were infused when the tumor reached $6 \mathrm{~mm}$ in diameter. In mice without tumors, labeled cells were infused i.v. 6-15 min after VEGF treatment. Five and $24 \mathrm{~h}$ after cancer cell infusion, the lungs were perfused with PBS under physical pressure to remove any circulating tumor cells and were then excised. Three lung tissue fragments ( $3 \mathrm{~mm}$ in diameter) were randomly selected and two $50-\mu \mathrm{M}$ sections per fragment were examined with a confocal microscope. The number of labeled cancer cells was normalized by total tissue surface area.

Metastasis assay in vivo. $4 \mathrm{~T} 1$ cells $\left(1 \times 10^{5}\right)$ labeled with DiI were intravenously injected at 2 weeks after the subcutaneous implantation of tumor cells $\left(2 \times 10^{6}\right)$ into the mice. Two weeks after injection, animals were sacrificed and lungs were resected. Surface metastasis was examined with light microscopy and further identification was performed with scanning for DiI-positive metastasis and processed for histological analysis. The metastasis was quantified as the total number of metastatic foci per reference area.

Immunofluorescence of lung tissue and MPVECs. The method of MPVEC isolation was described previously (2). MPVECs were cultured in a 24-well plate with various concentrations of VEGF for $4 \mathrm{~h}$ and then fixed by $4 \%$ paraformaldehyde. After washing with PBS, cells were incubated with $3 \%$ bovine serum albumin at room temperature for $30 \mathrm{~min}$, followed by incubation of celecoxib at a dilution of $1: 100$ overnight at $4^{\circ} \mathrm{C}$. For the immunofluorescence of lung tissue, the procedures of the first day were described above. Similarly, an FITC-conjugated fluorescent secondary antibody was added on the next day, and cells (or tissue sections) were detected under an inverted fluorescent microscope.

Tumor cell-MPVEC adhesion assay. MPVECs were cultured in 24-well plates with various concentrations of VEGF for $6 \mathrm{~h}$. 4T1 cells were suspended at a concentration of $1 \times 10^{6}$ cells $/ \mathrm{ml}$ in DMEM and labeled with 2',7'-bis-5-carboxyfluorescetin acetoxymethyl ester (BCECF, AM) at $37^{\circ} \mathrm{C}$ for $15 \mathrm{~min}$. Then, the labeled 4T1 cells were added to the MPVEC 24-well plates, and incubated at $37^{\circ} \mathrm{C}$ for $30 \mathrm{~min}$. After washing 3 times with PBS to remove unattached cells, the number of adhered AM-labeled 4T1 cells was counted using an inverted fluorescent microscope.

Measurement of circulating cytokines. The RayBio Custom mouse cytokines antibody array kit was purchased from RayBiotech and used according to the manufacturer's instructions. Briefly, after blocking with $1 \mathrm{X}$ blocking buffer, membranes were incubated for $1.5 \mathrm{~h}$ with the experimental serum. The membranes were washed and incubated with biotin-conjugated antibodies for $1.5 \mathrm{~h}$. The membranes were washed again and incubated with streptavidin-conjugated horseradish peroxidase for $2 \mathrm{~h}$, washed, and developed using an enhanced chemiluminescent substrate for horseradish peroxidase. Chemiluminescence was detected using EpiChemi $3^{\circledR}$ Darkroom imaging system and LabWorks ${ }^{\circledR}$ densitometry software. Data was corrected for background signal and normalized to positive controls using RayBio ${ }^{\circledR}$ Analysis Tool software (UVP Bioimaging, Upland, CA, USA).

Statistical analyses. The data were analyzed using JMP ver. 6 . The groups were compared using Chi-square test and Fisher's exact test. The analyses yielded $95 \%$ confidence intervals and 
A

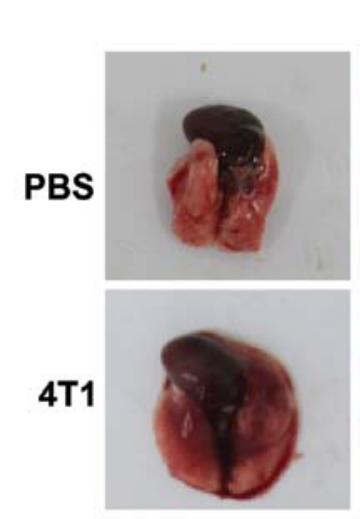

$\mathrm{He}$

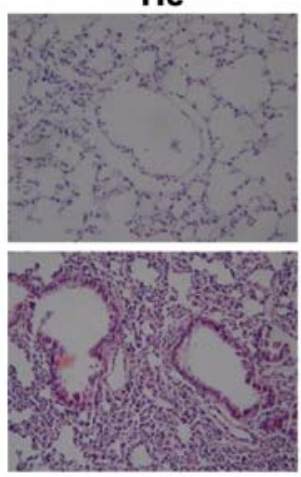

CD31

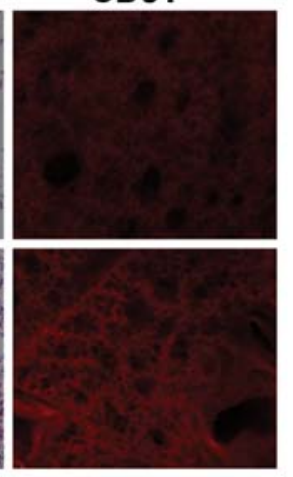

B

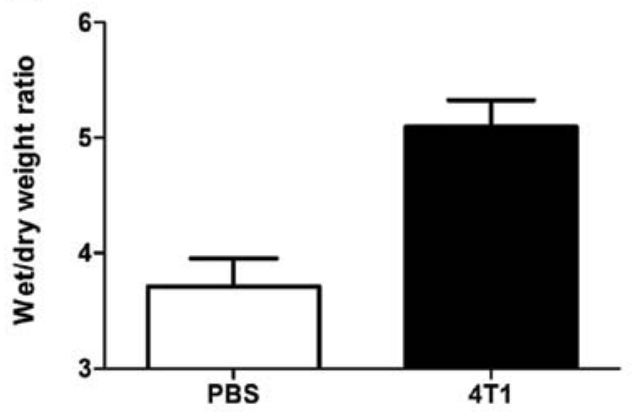

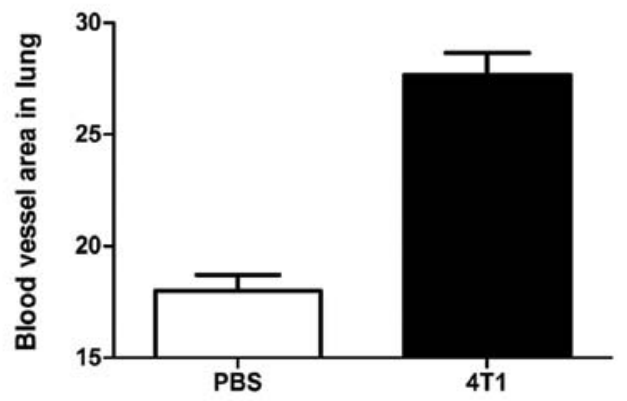

Figure 1. Inflammatory response in pre-metastatic lung induced by primary tumors. (A) Lungs from PBS-treated or tumor-bearing mice after 14 days. H\&E-stained lung section and CD31-stained lung section in PBS-treated mice. (B) Wet/dry-weight ratio of lungs and blood vessel area in lung.

P-values. Values of $\mathrm{P}<0.05$ were considered to indicate a statistically significant difference.

\section{Results}

Primary breast cancer tumor cells stimulate inflammation and BMDC recruitment in pre-metastatic lungs. We examined the effects of breast cancer-cell induction during the pre-metastatic phase on the morphology of lung tissues excised from 4T1 tumor-bearing mice. Edema and lung wet/ dry-weight ratios were significantly increased by breast cancer cell induction, compared with controls (Fig. 1). Hematoxylin and eosin (H\&E) staining also showed a higher level of inflammatory cell infiltration in the lungs of tumor-bearing mice, compared with non-tumor-bearing mice (Fig. 1A).

Immunohistochemical analysis with CD31 antibody showed that blood vessels in the lungs of 4T1-tumor-bearing mice appeared as primitive and dilated sinusoidal vascular structures, consisting of disorganized, tortuous, and interconnected vascular plexuses. Total blood vessel density was markedly increased in the lungs of tumor-bearing mice compared with non-tumor-bearing mice (Fig. 1A and B).

Metastatic breast cancer cells preferentially home to BMDC recruitment sites in pre-metastatic mouse lungs. During tumor progression, BMDCs have been shown to promote inappropriate growth, invasion and ultimately metastasis. In the present study, BMDC mobilization and recruitment changed in response to breast cancer cell inoculation. Green fluorescent protein (GFP) was used to label BMDCs and pre-metastatic lung fluorescence was detected by confocal microscopy following 4T1-tumor-cell injection in mice. Mice inoculated with PBS were used as controls. No $\mathrm{GFP}^{+} \mathrm{BMDCs}$ were observed in lungs without tumor cell injection (Fig. 2A), but $\mathrm{GFP}^{+} \mathrm{BMDCs}$ were found scattered in lung tissue by day 7 after tumor implantation in tumor-bearing mice. No BMDCs were observed in control lungs at the same stage. The difference increased at day 14 , when clusters of $\mathrm{GFP}^{+}$BMDCs were detected in lung tissues in tumor-bearing mice and near terminal bronchioles, while minimal $\mathrm{GFP}^{+} \mathrm{BMDCs}$ were observed in lung tissues in a small number of control mice (Fig. 2A and B).

The homing of tumor cells and their subsequent survival are critical for metastasis. To investigate the effects of BMDC recruitment on breast cancer cell homing in mouse lungs, DiI-labeled 4T1 cells were injected into mice 2 weeks after tumor inoculation. Clusters of $\mathrm{GFP}^{+} \mathrm{BMDCs}$ were detected near terminal bronchioles in 4T1-tumor-bearing mice (Fig. 2C). A subsequent metastasis assay revealed that the number of metastatic foci in lung lesions in 4T1-tumorbearing mice was significantly higher than that in the lungs of non-tumor-bearing mice (Fig. 2C and D).

Primary breast cancer cells trigger BMDC recruitment and inflammation in lungs through VEGF. We investigated the factor secreted by breast cancer cells responsible for inducing the pre-metastatic niche and BMDC recruitment. Mouse sera and 4T1 cell culture media were assayed using a RayBio Custom mouse cytokine antibody array kit. As shown in Fig. 3A, levels of VEGF, granulocyte-macrophage 

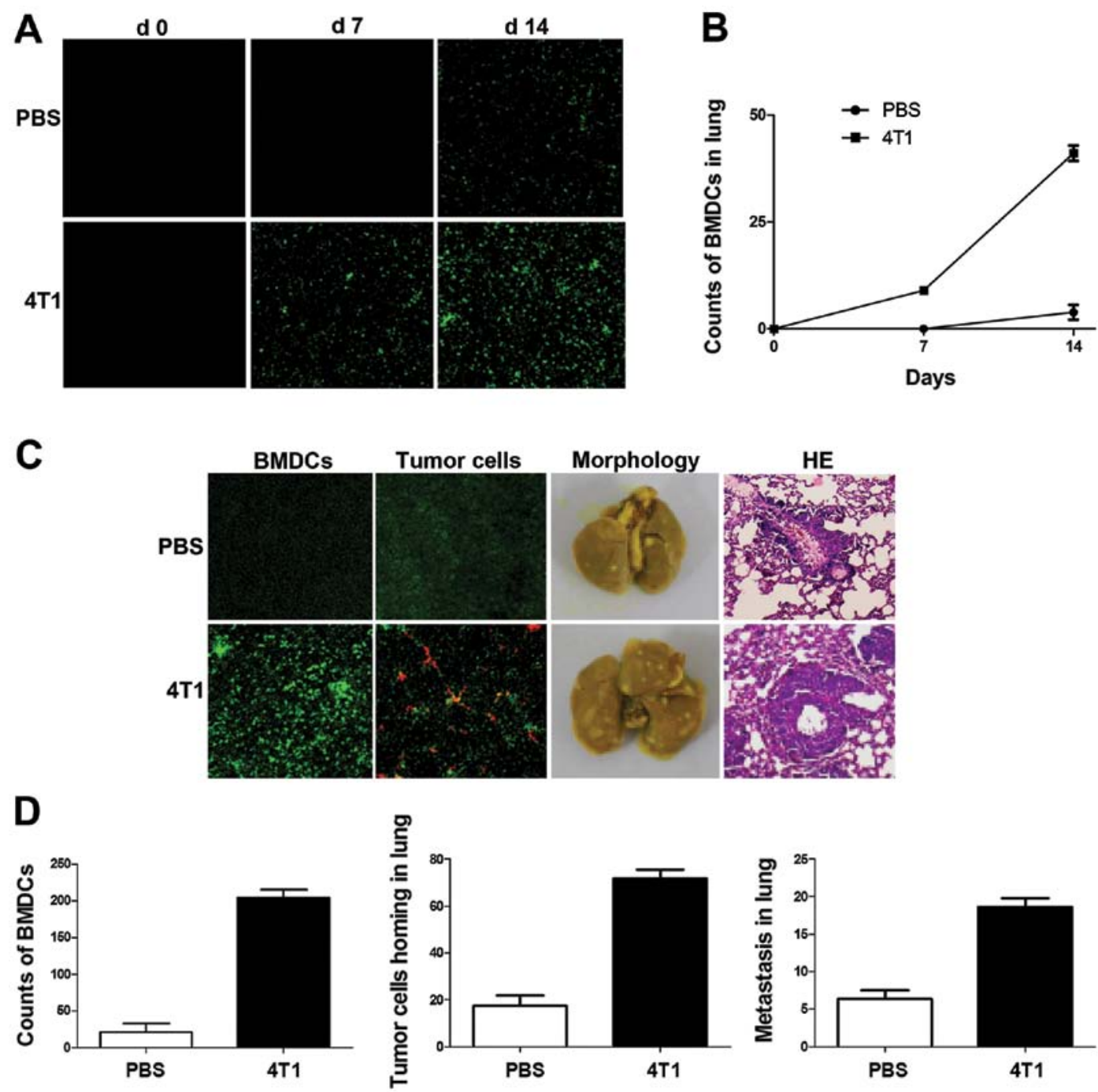

Figure 2. Metastatic cancer cells preferentially home to BMDC cluster sites in pre-metastatic lungs. (A) No BMDCs were observed in lungs of control mice on day 0,7 or 14. No BMDCs were observed in lungs of 4T1-tumor-bearing mice on day 0, some BMDCs were observed on day 7, and clusters of BMDCs were observed in lungs of 4T1-tumor-bearing mice on day 14. (B) Number of BMDCs in lungs. (C) GFP+ BMDCs were rarely observed in lungs of control mice compared with 4T1-tumor-bearing mice. Metastases were rare in lungs of control mice compared with 4T1 tumor-bearing mice. Clusters of GFP ${ }^{+}$ BMDCs (green) and tumor metastases (red) were detected. (D) Number of BMDCs, homing tumor cells and metastases.

colony-stimulating factor (GM-CSF) and interleukin-6 (IL-6) were significantly increased in sera from 4T1-tumor-bearing mice. However, only VEGF was detected in culture media from $4 \mathrm{~T} 1$ cells, at levels as high as $20 \mathrm{pg} / \mathrm{ml}$ (Fig. 3B); levels of GM-CSF, IL-6 and tumor necrosis factor- $\alpha$ (TNF $\alpha$ ) were too low for detection, suggesting that 4T1 cell-derived VEGF may be the critical triggering molecule responsible for initiating the formation of the pre-metastatic niche.

To investigate the role of VEGF in the initiation of the pre-metastatic niche, mice were treated daily with recombinant VEGF for 7 consecutive days and BMDC recruitment in the lung was tested. As expected, the frequency of BMDCs within the lungs, as well as angiogenesis, increased significantly in response to VEGF (Fig. 3C). To determine the effect of VEGF on the metastatic potential of breast cancer cells, mice were treated with VEGF for 7 consecutive days, and 4T1 cells were then injected through the tail vein. The number of visible metastatic foci in lungs revealed increased metastases (Fig. 3C and D), and examination of the lungs showed enlarged lung masses in VEGF-treated mice, compared with controls. These results suggest that VEGF is a crucial triggering molecule initiating the formation of the pre-metastatic niche.

Primary tumors induce PGE2 production and inflammatory response in pre-metastatic lungs. The important role of PGE2 in tumor angiogenesis and metastasis suggests that its expression may be correlated with VEGF. We therefore compared PGE2 levels, and levels of its synthesis regulator cyclooxygenase-2 (COX-2) gene, in pre-metastatic lungs in mice with and without 4T1 tumors. As shown in Fig. 4, COX-2 expression in lung tissues was upregulated at both mRNA and protein levels by inoculation of 4T1 cells, and the increase in PGE2 production confirmed the COX-2-expression results.

Notably, the expression profiles in MPVECs induced by VEGF and PGE2 were similar. Among the genes with $>2$-fold changes in expression in response to stimulation, 48 genes were upregulated and 35 were downregulated in response to VEGF, and 65 and 33, respectively, in response to PGE2. 

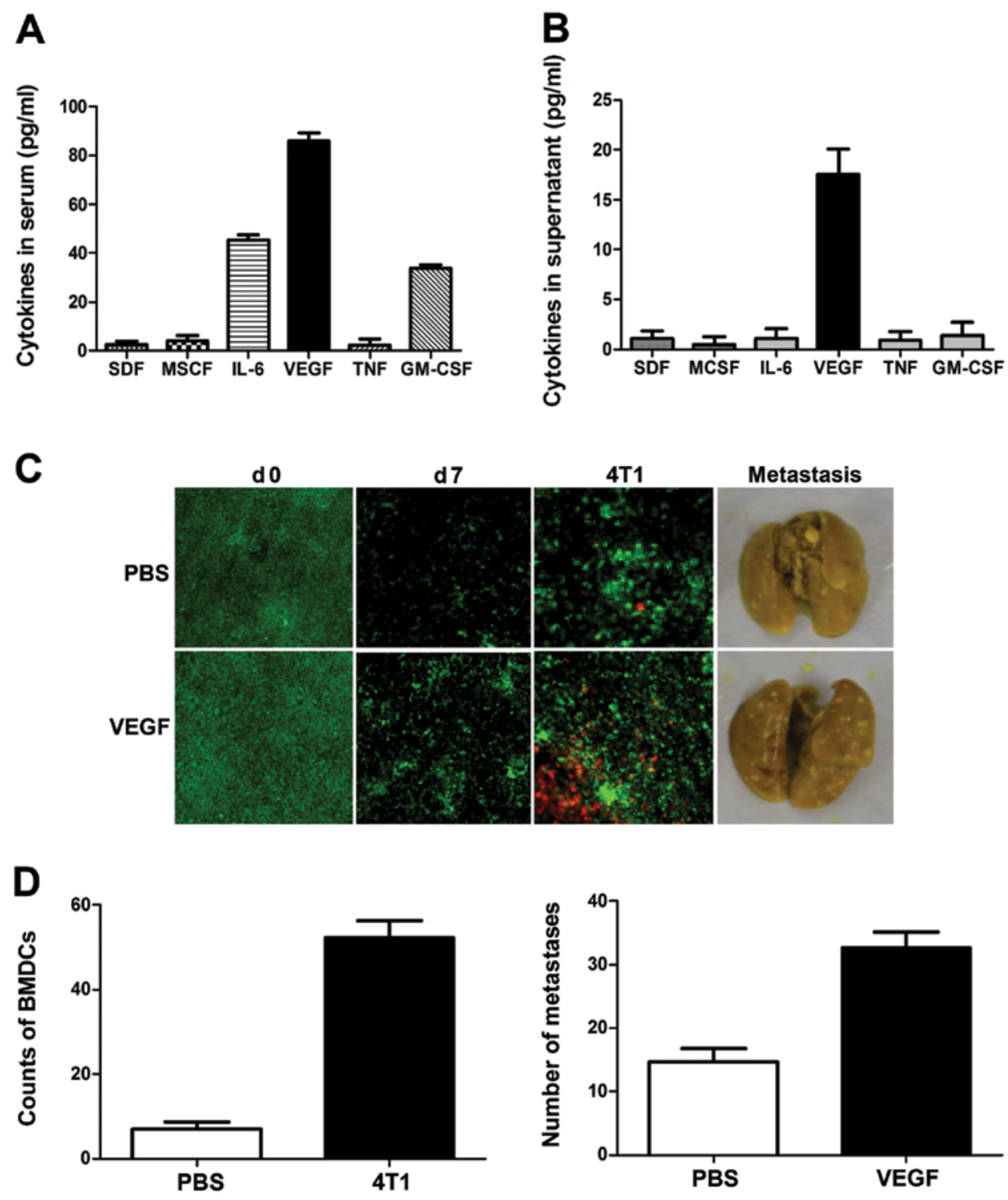

Figure 3. Pretreatment with VEGF induces BMDC migration to the lungs and promotes metastasis. (A and B) Cytokine levels in serum (A) and supernatant (B). (C) DiI-labeled metastatic 4T1 cells (red) and BMDCs (green) homing in lungs of PBS-treated mice, and gross metastasis in lungs of PBS-treated mice. (D) Number of BMDCs and metastases.

A PBS 4T1
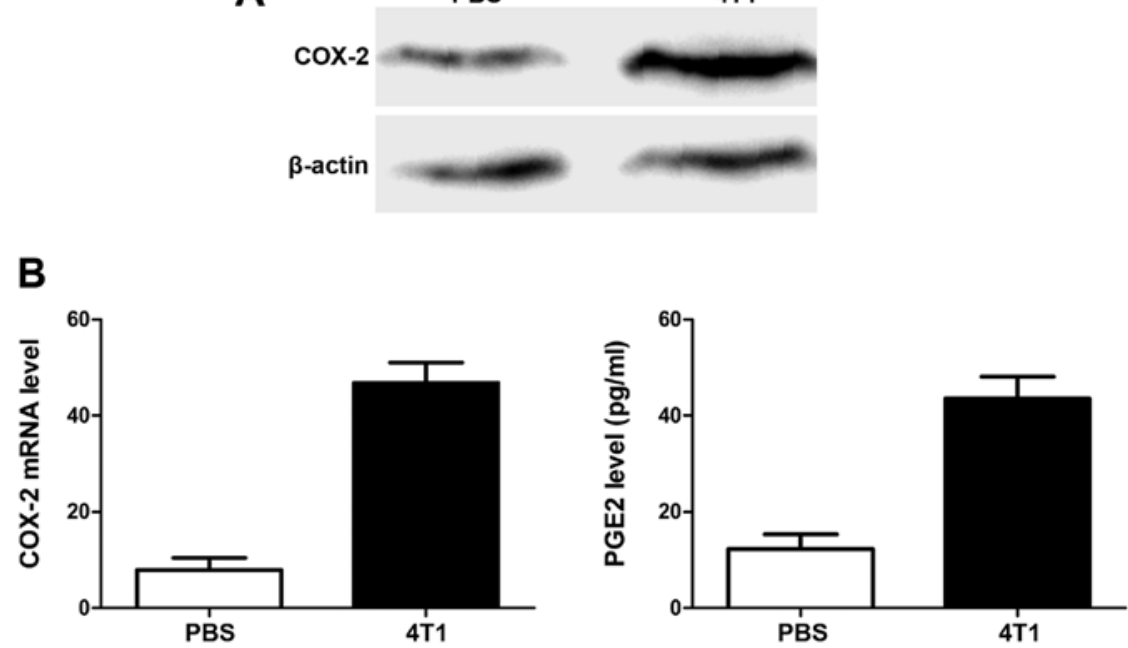

Figure 4. PGE2 and COX-2 expression levels. (A) COX-2 expression was examined by western blotting in pre-metastatic lungs of mice bearing 4T1 tumors and control mice. (B) qRT-PCR analysis of COX-2 expression and PGE2 levels was examined by ELISA. 


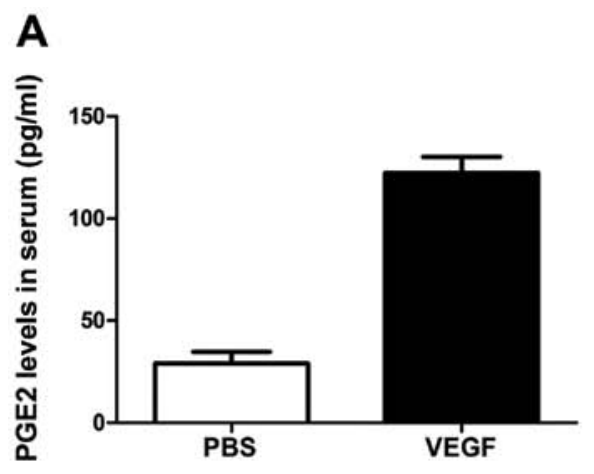

B

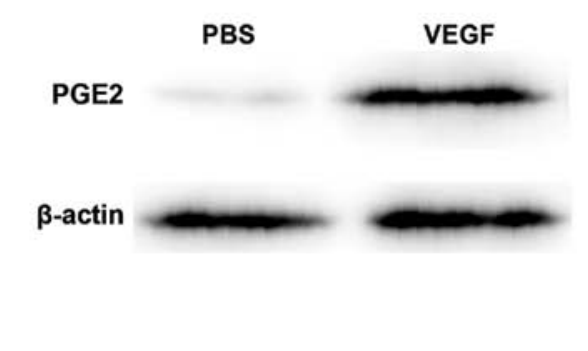

\section{C}

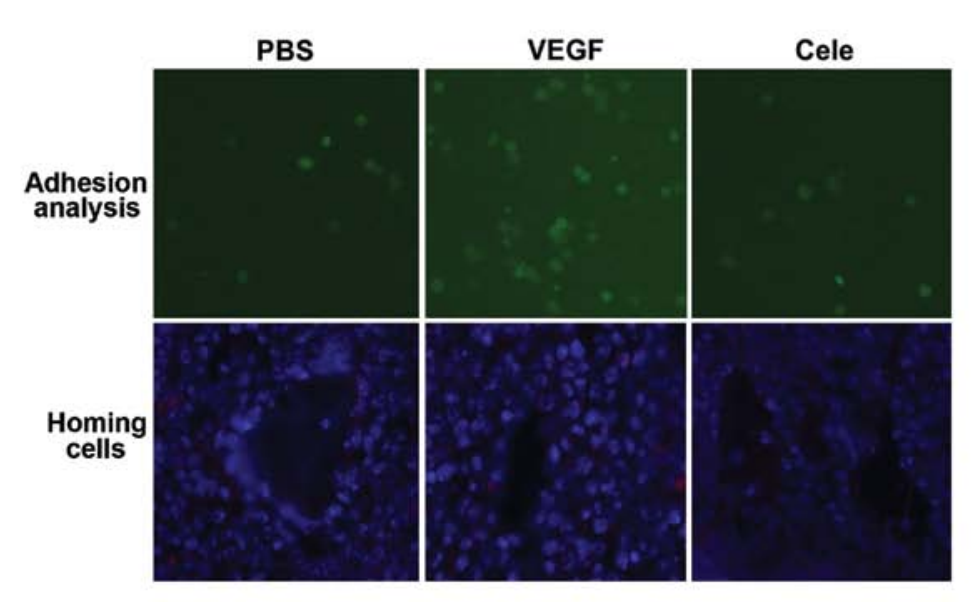

D
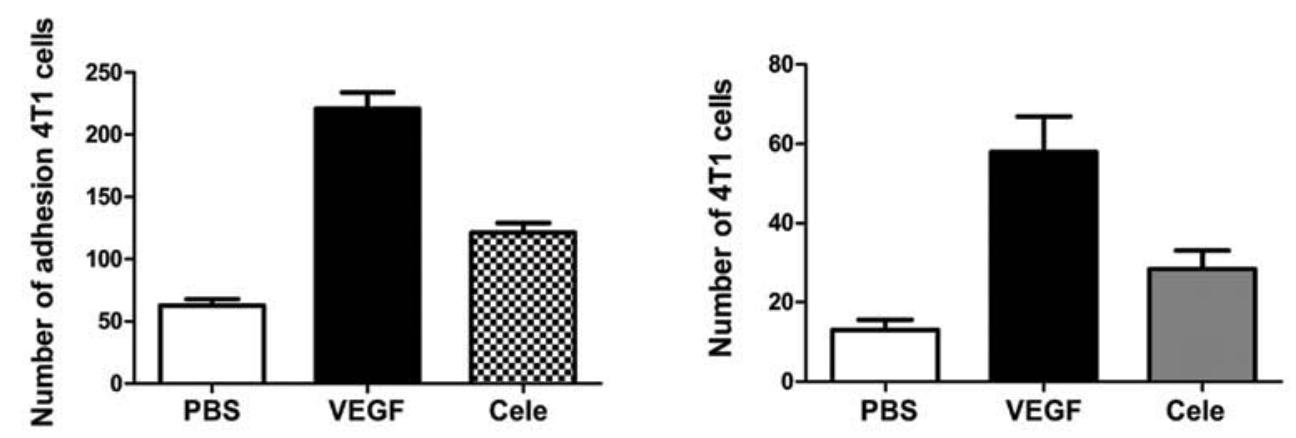

Figure 5. VEGF mediates 4T1 cell homing in lungs via PGE2. (A and B) PGE2 levels in serum and in cells. (C) Tumor cell MPVEC adhesion assays for cells treated with PBS, VEGF $(5 \mu \mathrm{g} / \mathrm{ml})$ and celecoxib. VEGF-stimulated tumor cell homing in lungs was significantly reduced by treatment with celecoxib. (D) Number of adhering tumor cells and 4T1 cells homing in lungs.

Notably, 33 upregulated genes and 14 downregulated genes were common to both responses, suggesting that they used the same downstream pathways.

VEGF plays key roles in inducing pre-metastatic recruitment of BMDCs in lungs and its function is suppressed by COX-2 inhibition. PGE2 is a secreted protein previously characterized as a proinflammatory factor. We defined the role of PGE2 in VEGF-induced BMDC recruitment and lung metastasis in primary mouse lung endothelial cells exposed to VEGF. As expected, VEGF stimulation significantly increased COX-2 expression and PGE2 levels in endothelial cells (Fig. 5A and B). We also evaluated the role of PGE2 induction by VEGF in endothelial cell adhesion to metastatic cancer cells in an in vitro cancer cell adhesion assay. VEGF stimulation increased the number of fluorescently-labeled metastatic cancer cells attached to lung endothelial cells (Fig. 5C and D). The addition of celecoxib abolished tumor cell adhesion to the endothelial cells (Fig. 5C and D). PGE2 overexpression resulted in a significant increase in the homing of infused metastatic cancer cells to the lungs of mice treated with VEGF (Fig. 5C and D), measured $48 \mathrm{~h}$ after metastatic cancer cell infusion (Fig. 5C and D). Collectively, these data show that blocking lung endothelial PGE2 production reduced VEGF-induced breast cancer metastasis to the lungs.

VEGF mediates $4 T 1$ cell homing to the lungs via COX-2. The role of COX-2 in VEGF-induced BMDC recruitment and lung metastasis was defined by treating mice with VEGF and the COX-2 inhibitor, celecoxib. Celecoxib treatment reduced the 
A
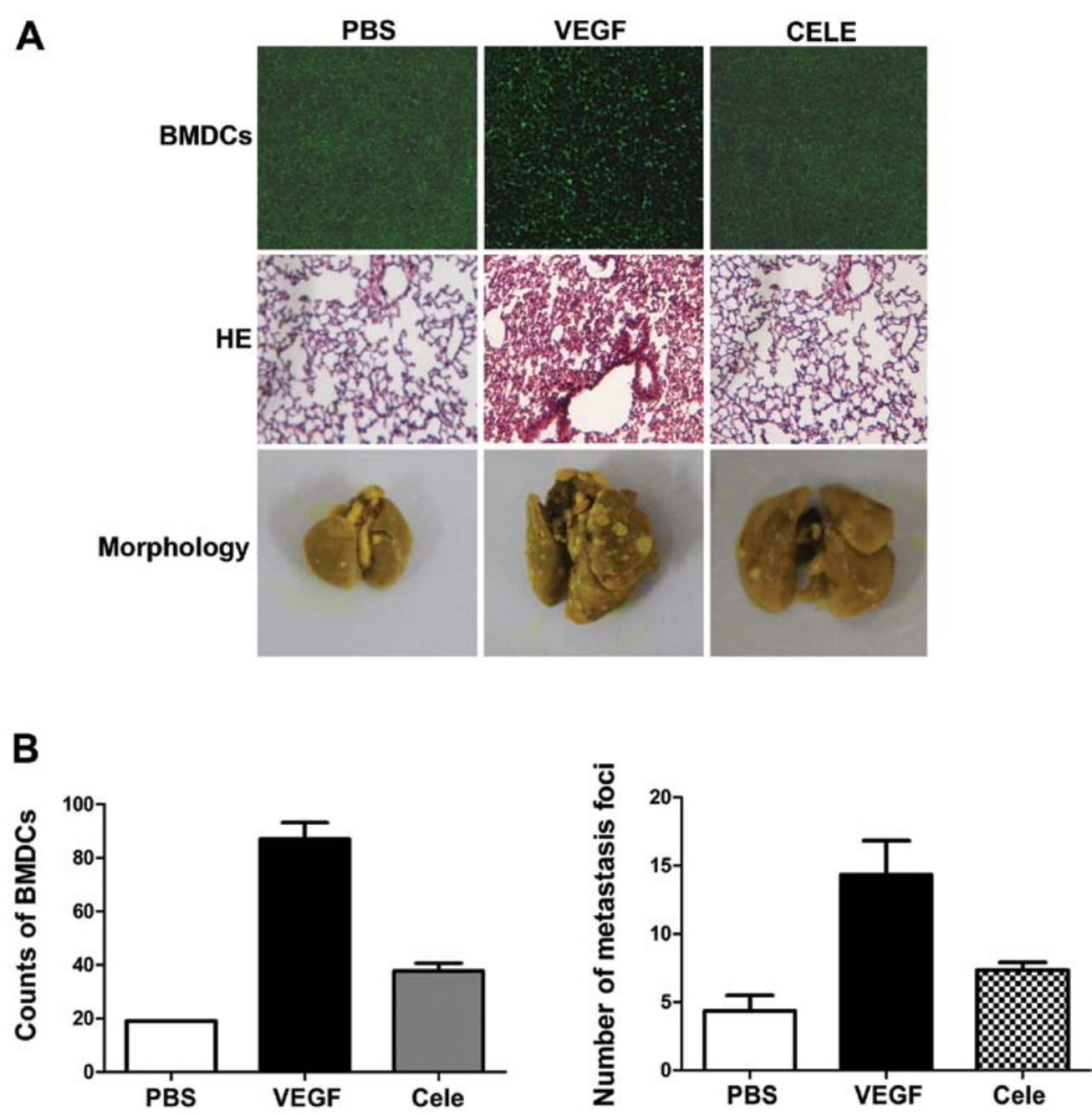

Figure 6. Celecoxib treatment reduces lung inflammation and metastasis. (A) $\mathrm{GFP}^{+}$bone marrow cells in lungs of PBS, VEGF and celecoxib-treated mice; H\&E-stained lung section from PBS, VEGF and celecoxib-treated mice; lung metastasis in PBS, VEGF and celecoxib-treated mice. (B) Number of BMDCs and metastasis foci per mouse.

recruitment of BMDCs in lungs (Fig. 6A and B), and lung inflammation was also reduced, as shown by H\&E staining (Fig. 6A). 4T1 cells were then injected into the tail vein of mice pretreated with VEGF in the presence or absence of celecoxib. Celecoxib treatment significantly reduced VEGF-induced lung metastases of 4T1 cells (Fig. 6A and B). These results indicate that PGE2 is involved in VEGF-induced pre-metastatic microenvironmental changes.

\section{Discussion}

Some researchers have suggested that therapy for metastasis should be targeted not only against tumor cells, but also against the host microenvironment that contributes to and supports the progressive growth and survival of metastatic cancer cells (13-18). The results of this study showed that BMDC recruitment and inflammatory responses could be induced in pre-metastatic lungs by distant 4T1 breast cancer in mice, and that pre-metastatic changes contributed to the homing of metastatic cells in lungs. The process of cancer metastasis is complex and consists of a series of inter-related steps. Extravasation and homing of metastatic tumor cells in specific organs is a checkpoint in the production of clinically- relevant lesions. Previous studies have suggested that primary tumors are able to modify the distant microenvironment before the arrival of metastatic tumor cells, to create what is known as the pre-metastatic niche (19-23). This ability of tumors to affect distant tissues is expected to enable cancer cells to target specific organs, and BMDCs are thought to be major players in these processes. In accordance with these reports, our results showed that clusters of $\mathrm{GFP}^{+}$BMDCs and an inflammatory response were detected in lung tissues by day 14 after tumor implantation.

To determine which factor(s) secreted by tumor cells might be responsible for mobilizing BMDCs, we measured plasma and tumor levels of several factors previously implicated in BMDC mobilization. However, only VEGF correlated with the ability of tumors to metastasize, and pretreatment with VEGF was sufficient to mimic the pre-metastatic environment initiated by primary tumors. Previous studies have shown that multiple secreted factors overexpressed in primary tumors, including VEGF, transforming growth factor- $\beta$, TNF- $\alpha$, and angiopoietin-2, can induce lung microenvironmental changes and enhance the metastatic properties of several tumors. VEGF released from lung-metastasizing cancer cells can activate the Src-FAK complex in lung endothelial cells and promote 
vascular hyperpermeability, upregulation of endothelial adhesion molecules, and cancer-cell homing. We demonstrated that primary tumor-derived VEGF was involved in induction of the inflammatory response in pre-metastatic lungs.

Our data indicated that tumor-secreted VEGF induced PGE2 expression in pre-metastatic lungs. Treatment with celecoxib significantly reduced the number of VEGF-induced metastases, emphasizing the important role of BMDC homing in the lung. Our results suggest that anti-inflammatory agents may modulate the microenvironment in target organs through suppression of the inflammatory response, leading to marked inhibition of lung metastasis, which may be an alternative mechanism of action for these agents.

The mechanism by which celecoxib modulates the microenviroment in the lung remains to be elucidated. PGE2 expression is rapidly induced in response to inflammatory stimuli, such as TNF- $\alpha$ or lipopolysaccharide $(24,25)$. VEGF overexpression has been reported to lead to PGE2 production $(26,27)$, while exposure of cultured endothelial cells to tumorsecreted factors increased PGE2 expression (28-30), and VEGF directly induced PGE2 expression in endothelial cells (31). We showed that PGE2 can function as a chemoattractant to enhance the mobilization of BMDCs and facilitate their homing into the lung, before the arrival of tumor cells. Lung regions thus provide discrete, fertile fields of pre-metastatic 'soil' where increased tumor-cell-homing is facilitated by the secretion of factors by endothelial cells.

In summary, our results provide direct evidence for the ability of primary tumor-derived VEGF to trigger a host inflammatory response in the lung microenvironment through PGE2 production, leading circulating tumor cells to localize preferentially in regions of high BMDC recruitment. These results are in agreement with those of other studies that have implicated the proinflammatory host response in promoting the process of lung metastasis.

\section{Acknowledgements}

We gratefully acknowledge the financial support from the $\mathrm{Ph}$.D. Programs Foundation of Ministry of Education of China (20120131120046), the Natural Science Foundation (General programs 81272351), the Natural Science Foundation of Shandong Province (ZR2012HM020, BS2010YY038), and the Key Development Program for Basic Research of Shandong Province (2012G0021826).

\section{References}

1. Nguyen DX, Bos PD and Massagué J: Metastasis: from dissemination to organ-specific colonization. Nat Rev Cancer 9: 274-284, 2009.

2. Fidler IJ: The organ microenvironment and cancer metastasis. Differentiation 70: 498-505, 2002.

3. Das Roy L, Pathangey LB, Tinder TL, Schettini JL, Gruber HE and Mukherjee P: Breast-cancer-associated metastasis is significantly increased in a model of autoimmune arthritis. Breast Cancer Res 11: Jul 30, 2009 (Epub ahead of print). doi: 10.1186/ bcr2345.

4. Hiratsuka S, Watanabe A, Aburatani H and Maru Y: Tumourmediated upregulation of chemoattractants and recruitment of myeloid cells predetermines lung metastasis. Nat Cell Biol 8: 1369-1375, 2006.
5. Kaplan RN, Riba RD, Zacharoulis S, et al: VEGFR1-positive haematopoietic bone marrow progenitors initiate the pre-metastatic niche. Nature 438: 820-827, 2005.

6. Hanahan D and Weinberg RA: The hallmarks of cancer. Cell 100: 57-70, 2000.

7. Chen SF, Fei X and Li SH: A new simple method for isolation of microvascular endothelial cells avoiding both chemical and mechanical injuries. Microvasc Res 50: 119-128, 1995.

8. Lu H, Ouyang W and Huang C: Inflammation, a key event in cancer development. Mol Cancer Res 4: 221-233, 2006.

9. Fidler IJ: The pathogenesis of cancer metastasis: the 'seed and soil' hypothesis revisited. Nat Rev Cancer 3: 453-458, 2003.

10. Murdoch C, Muthana M, Coffelt SB and Lewis CE: The role of myeloid cells in the promotion of tumour angiogenesis. Nat Rev Cancer 8: 618-631, 2008.

11. Kim S, Takahashi H, Lin WW, et al: Carcinoma-produced factors activate myeloid cells through TLR2 to stimulate metastasis. Nature 457: 102-106, 2009.

12. Carmeliet P: VEGF as a key mediator of angiogenesis in cancer. Oncology 69: 4-10, 2005.

13. Mantovani A, Allavena P, Sica A and Balkwill F: Cancer-related inflammation. Nature 454: 436-444, 2008.

14. Padua D, Zhang XH and Wang Q: TGFbeta primes breast tumors for lung metastasis seeding through angiopoietin-like 4. Cell 133: 66-77, 2008.

15. Serafini P, Borrello I and Bronte V: Myeloid suppressor cells in cancer: recruitment, phenotype, properties, and mechanisms of immune suppression. Semin Cancer Biol 16: 53-65, 2006.

16. Yang L, Huang J, Ren X, et al: Abrogation of TGFbeta signaling in mammary carcinomas recruits $\mathrm{Gr}-1^{+} \mathrm{CD} 11 \mathrm{~b}^{+}$myeloid cells that promote metastasis. Cancer Cell 13: 23-35, 2008.

17. Olkhanud $\mathrm{PB}$, Baatar D, Bodogai $\mathrm{M}$, et al: Breast cancer lung metastasis requires expression of chemokine receptor CCR4 and regulatory T cells. Cancer Res 69: 5996-6004, 2009.

18. Huang Y, Song N, Ding Y, et al: Pulmonary vascular destabilization in the premetastatic phase facilitates lung metastasis. Cancer Res 69: 7529-7537, 2009.

19. Kitamura T, Kometani K, Hashida H, et al: SMAD4-deficient intestinal tumors recruit $\mathrm{CCR} 1^{+}$myeloid cells that promote invasion. Nat Genet 39: 467-475, 2007.

20. Dawson MR, Duda DG, Fukumura D and Jain RK: VEGFR1activity independent metastasis formation. Nature 461: E4-E5, 2009.

21. Erler JT, Bennewith KL, Cox TR, et al: Hypoxia-induced lysyl oxidase is a critical mediator of bone marrow cell recruitment to form the premetastatic niche. Cancer Cell 15: 35-44, 2009.

22. Duda DG, Cohen KS, Kozin SV, et al: Evidence for incorporation of bone marrow-derived endothelial cells into perfused blood vessels in tumors. Blood 107: 2774-2776, 2006.

23. Dawson MR, Duda DG, Chae SS, Fukumura D and Jain RK: VEGFR1 activity modulates myeloid cell infiltration in growing lung metastases but is not required for spontaneous metastasis formation. PLoS One 4: e6525, 2009.

24. Bos CL, Richel DJ, Ritsema T, et al: Prostanoids and prostanoid receptors in signal transduction. Int J Biochem Cell Biol 36: 1187-1205, 2004.

25. Ma X, Kundu N, Ioffe OB, et al: Prostaglandin E receptor EP1 suppresses breast cancer metastasis and is linked to survival differences and cancer disparities. Mol Cancer Res 8: 1310-1318, 2010.

26. Narumiya S, Sugimoto Y and Ushikubi F: Prostanoid receptors: structures, properties, and functions. Physiol Rev 79: 1193-1226, 1999.

27. Yang T and Du Y: Distinct roles of central and peripheral prostaglandin E2 and EP subtypes in blood pressure regulation. Am J Hypertens 25: 1042-1049, 2012.

28. Gupta GP, Nguyen DX, Chiang AC, et al: Mediators of vascular remodeling co-opted for sequential steps in lung metastasis. Nature 446: 765-770, 2007.

29. Nozawa H,Chiu C and Hanahan D: Infiltrating neutrophils mediate the initial angiogenic switch in a mouse model of multistage carcinogenesis. Proc Natl Acad Sci USA 103: 12493-12498, 2006.

30. Fridlender ZG, Sun J, Kim S, Kapoor V, Cheng G, Ling L, et al: Polarization of tumor-associated neutrophil (TAN) phenotype by TGF-beta: 'N1' versus 'N2' TAN. Cancer Cell 16: 183-194, 2009.

31. Chang SH, Liu CH, Conway R, et al: Role of prostaglandin E2-dependent angiogenic switch in cyclooxygenase 2-induced breast cancer progression. Proc Natl Acad Sci USA 101: 591-596, 2004. 